\title{
Subacute myelopathy caused by spinal venous infarction
}

\author{
C.E. Clarke and W.J. K. Cumming \\ Department of Neurology, University Hospital of South Manchester, West Didsbury, Manchester, M20 8LR, UK.
}

\begin{abstract}
Summary: A 44 year old female presented with a subacute myelopathy in association with pelvic venous thrombosis. It is inferred from the temporal relationship of these events that the patient suffered a subacute spinal venous infarction. This is discussed along with the aetiology, anatomical distribution and management of the condition.
\end{abstract}

\section{Introduction}

The syndrome of acute or subacute transverse myelopathy (ATM) was first recognized by Foix \& Alajouanine in $1926 .{ }^{1}$ Although it has been associated with many conditions, ${ }^{2}$ detailed neurophysiological, neuroradiological and pathological investigations have, thus far, failed to advance our knowledge with regard to its aetiology. Early pathological studies identified thrombosis in the spinal venous channels as a possible cause, but confirmation of this at postmortem has only been documented in 15 patients according to a recent review. ${ }^{3}$ The latter authors suggested that subacute and chronic spinal venous infarction may be responsible for spinal cord syndromes which have hitherto been labelled as idiopathic.

We describe a patient in whom a subacute myelopathy developed in association with radiologically proven pelvic venous thrombosis. The possibility of spinal venous infarction in the case is discussed, together with its aetiology, anatomical distribution and treatment.

\section{Case report}

A 44 year old female suddenly became aware of paraesthesiae spreading upward from the toes to the level of the groins whilst in the bath. This was associated with considerable pain in the same distribution which she described as having a burning dysaesthetic quality. She was unable to get out of the bath because of lower limb weakness and over the next few hours, her legs developed a mottled cyanotic discolouration.

Over the next few days, the power in her lower limbs gradually improved but she continued to suffer paras-

Correspondence: W. J. K. Cumming B.Sc., M.D., F.R.C.P.I. Accepted: 11 February 1987 thesiae and pain. Her legs remained slightly discoloured and this became worse if she adopted the upright posture for any length of time. At this stage, bladder and bowel function were normal.

Hypercholesterolaemia had been found several years earlier during the investigation of xanthelasmata but there was no other significant past history. She smoked 30 cigarettes daily but had never received an oral contraceptive preparation and denied any recent period of immobilization.

Examination 15 days after the onset of the illness revealed a spastic paraparesis with diminished spinothalamic sensation to the T12 dermatome with preservation of posterior column function. No abnormalities were found in the upper limbs or cranial nerve territory and higher mental functions were preserved. Both lower limbs had a dusky cyanotic hue but the peripheral pulses were all present and symmetrical. She was in sinus rhythm and normotensive with no vascular or cardiac bruits. Abdominal examination was also unremarkable.

A full blood count, platelet count, erythrocyte sedimentation rate, biochemical profile, thyroid function tests, myeloma and lupus screens and coagulation studies were all within normal limits. Fasting lipids confirmed a Fredrickson type IIb hyperlipidaemia serum cholesterol $7.6 \mathrm{mmol} / 1$ (normal 3.6-7.2) and serum triglyceride $2.3 \mathrm{mmol} / 1$ (normal $0.3-1.8$ ). A lumbar puncture produced clear colourless fluid under normal pressure with 10 red blood cells $/ \mathrm{ml}$ and 3 white blood cells $/ \mathrm{ml}$ and no bacterial growth. The cerebrospinal fluid protein was $0.47 \mathrm{~g} / 1$ (normal $0.25-0.45$ ) and glucose $3.3 \mathrm{mmol} / 1$ (normal 2.5-5.5).

Radiographic investigations were initiated with lower limb arteriography in view of the vascular picture. This failed to reveal any arterial occlusion and therefore plain radiology of the thoraco-lumbar spine was performed. This demonstrated spina bifida occulta at L 5 but pan-myelography did not locate any 


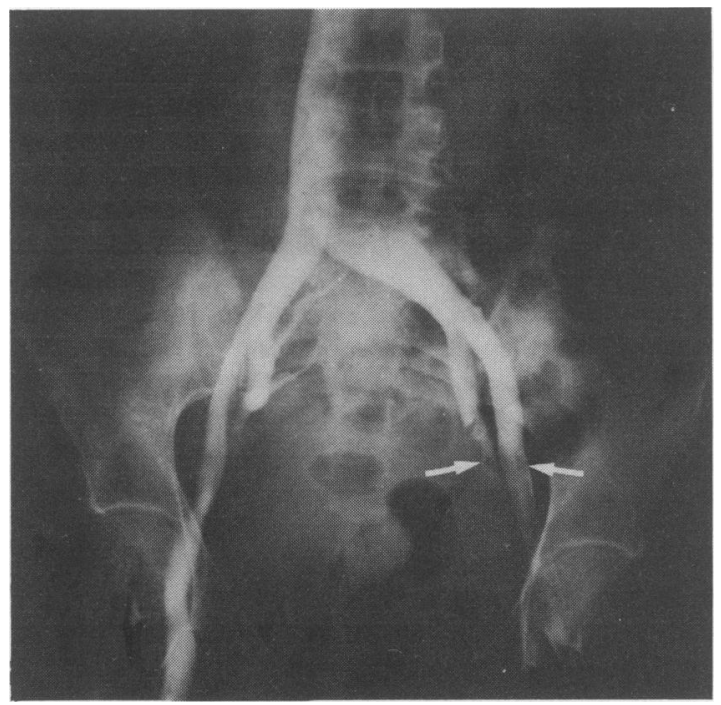

Figure 1 Lower limb venogram performed by simultaneous common femoral vein contrast injections. Note thrombus in the left common femoral and internal iliac veins (arrows).

abnormality at this or any other level.

Again returning to the possibility of a vascular lesion, venography of the lower limb vessels was performed by simultaneous contrast injections into the common femoral veins. This outlined filling defects in the left common femoral and left internal iliac veins (Figure 1), although the common iliac veins and inferior vena cava appeared normal. A chest Xray and technetium lung perfusion scan failed to reveal any evidence of occult pulmonary embolism and an abdominal computed tomographic scan did not identify any external compression of the affected vessels.

The patient was commenced on intravenous heparin following venography and subsequently controlled on oral nicoumalone therapy. Over the ensuing 6 months, lower limb spasticity increased in spite of baclofen therapy and she developed features of a neurogenic bladder.

\section{Discussion}

Acute spinal venous infarction is reputed to be rare, only 15 pathologically confirmed cases appearing in the literature according to a recent review. ${ }^{3}$ The latter authors believed that less devastating venous infarction may occur leading to some of the idiopathic subacute and chronic myelopathies. However, clinicopathological surveys of patients with transverse

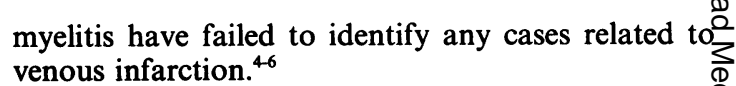

The spinal cord syndrome in our patient arose in association with venographically proven thrombus in: the left common femoral and internal iliac veins myelography, arteriography and cerebro-spinal fluido biochemistry were all normal. Since cyanotic discolouration of the legs was noticed at the outset of the illness, lower limb venous insufficiency must have been present at the onset of cord dysfunction. This suggests a causal link between the myelopathy and the venous abnormality, although when the venogram was per $\overrightarrow{0}$ formed several weeks later, no thrombus was seen in the inferior vena cava which might have accounted fof such an association. We therefore propose that this is a case of subacute spinal venous infarction, whils recognizing that pathological proof is necessarilys; lacking at present. Further investigations such as computed tomography or magnetic resonance imag ing may have demonstrated swelling and oedema of the spinal cord, but this would not have been specifie to a venous aetiology. Spinal angiography may alsoo have failed to confirm our proposition and may have been detrimental, vascular cord syndromes nots infrequently occurring as a consequence of the्छ procedure.

With regard to the aetiology of venous thrombosis in this case, none has been established. Possi@l $£_{0}^{\circ}$ contributory factors include the hyperlipidaemia a $\mathrm{d}^{\mathrm{N}}$ cigarette smoking. Previous reports of venous infa다음 tion of the cord have attributed the lesion to condis tions such as decompression sickness ${ }^{7}$ and fibro-car tilaginous emboli from degenerate inter-vertebraf discs. ${ }^{8}$ However, the most frequent cause appears to be venous thrombosis due to conditions such as severe sepsis, polycythaemia rubra vera and pancreatic car응 cinoma. $^{3}$

The anatomical distribution of infarction in thie case is particularly interesting. Both the lateral cor tico-spinal and the spino-thalamic tracts were involved, posterior column functions being preserved $\overline{-}$ This situation is analogous to that seen in the anteriop. spinal artery syndrome. Although this condition can not be ruled out in the present case, it seems more likely that venous thrombosis has occurred in the territory of the anterior spinal vein. Gillilan' has previously shown that the anterior segment of the spinal cord is drained by the anterior spinal vein in manner analogous to the anterior spinal artery. It is interesting to compare this with the work of Doppman and colleagues ${ }^{10}$ who failed to show any clinical effect after inducing thrombosis of the posterior spinal vein of the Rhesus monkey. Yet at necropsy, symmetricak white matter oedema, demyelination and gliosis hac occurred in the posterior columns of the treated animals.

Unfortunately, it is not possible to show more 
conclusively that our patient had suffered an episode of venous infarction of the cord. At present, such a diagnosis must remain a clinical one alone, although evidence of other venous abnormalities may suggest the diagnosis as in this case. If a more concrete diagnosis could be made, therapeutic intervention with thrombolytic agents such as streptokinase might be justified in an effort to regain some cord function. The former agents have already proven to be of significant benefit given intravenously in acute myocardial infarction. " Also, prophylactic anti-

\section{References}

1. Foix, C. \& Alajouanine, T. La myelite necrotique subaige. Rev Neurol 1926, 2: 1-42.

2. Editorial. Acute transverse myelopathy. Lancet 1986, i: 20-21.

3. Rao, K.R., Donnenfield, H., Chusid, J.G. \& Valdez, S. Acute myelopathy secondary to spinal venous infarction. J Neurol Sci 1982, 56, 107-113.

4. Altrocchi, P.H. Acute transverse myelopathy. Arch Neurol 1963, 9: 111-114.

5. Lipton, H.L. \& Teasdall, R.D. Acute transverse myelopathy in adults. Arch Neurol 1973, 28: 252-257.

6. Whiteley, A.M., Hauw, J.J. \& Escourolle, R. A pathological survey of 41 cases of acute intrinsic spinal cord disease. J Neurol Sci 1979, 42: 229-242. coagulation in these patients may prevent extension or recurrence of thrombosis. We therefore suggest that clinicians maintain a higher index of suspicion for venous abnormalities in patients with idiopathic cord syndromes so as not to miss this therapeutic potential.

\section{Acknowledgements}

The authors wish to thank the Department of Medical Illustration, University Hospital of South Manchester for the preparation of the photographic material.

7. Hallenbeck, J.M., Bove, A.A. \& Elliot, D.M. Mechanisms underlying spinal cord damage in decompression sickness. Neurology 1975, 25: 308-316.

8. Feigin, I., Popoff, N. \& Masazumi, A. Fibro-cartilagenous emboli to the spinal cord with necrotic myelopathy. $J$ Neuropath Exp Neurol 1965, 24: 63-74.

9. Gillilan, L.A. Veins of the spinal cord. Neurology 1970, 20: $860-868$.

10. Doppman, J.L., Girton, M. \& Popovsky, M.A. Acute occlusion of the posterior spinal vein. J Neurosurg 1979, 51: 201-205.

11. Editorial. Streptokinase in acute myocardial infarction. Lancet 1986, i: 421-422. 